\title{
Simultaneous Delivery of Wireless LAN and Cellular Radio Signals over Optical Fiber
}

\author{
Roland Yuen and Xavier N. Fernando, Senior Member, IEEE \\ Ryerson University, Toronto, Canada \\ xavier@ieee.org
}

\begin{abstract}
Recent boom in wireless hot-spots has triggered interest in optical fiber based wireless access schemes. An enhanced hot-spot will support both IEEE 802.11 wireless LAN and cellular radio services. Simultaneous transmission of these radio signals over a single radio-over-fiber (ROF) link is possible in a subcarrier multiplexed manner. However, link design in this multi-system scenario is a complex task. There are number of quality measures such as signal to noise, distortion and interference ratios involved both in the optical link and in the air interface. These are functions of several parameters such as the fiber length, bandwidth, modulation depth, radio cell size and relative $\mathrm{RF}$ power of both systems. In this paper we analyze the uplink and show a way to decide the cumulative optical modulation index $(\mu)$ and the RF power ratio $(T)$ that will yield the best performance for both systems.
\end{abstract}

\section{INTRODUCTION}

Recently, wireless hotspots have become the center of attraction due to low cost and high speed, however, the major issue is the limited coverage. In order to support large coverage area, many hotspots are required which is a technical and economical challenge [1]. On the other hand, next generation cellular networks supporting relatively low speed services, to large radio cells could be combined with WLAN hotspots [2]. This integration will provide network connectivity everywhere and high date rate in strategic locations.

In a cellular CDMA environment, it was shown that the cumulative SNR is the weighted sum of both optical and electrical SNRs, and smaller than the smallest [?]. Even sub-picometer filter to optically demultiplex $900 \mathrm{MHz}$ and $2.4 \mathrm{GHz} \mathrm{RF}$ signals within the fiber [3] has been developed. In this paper, the performance of a subcarrier multiplexed enhanced wireless hotspot that support both wideband code division multiplex access (WCDMA) cellular services at $900 \mathrm{MHz}$ and IEEE 802.11b WLAN services at $2.4 \mathrm{GHz}$ band is investigated.

Figure 1 illustrates the basic architecture of a micro cellular system employing radio-over-fiber ROF links. A central base station is linked to multiple radio access points (RAP) through ROF links. A RAP provides wireless services to many mobile stations (MSs). Several authors have investigated similar systems [4]. However, we differ that in our system, each RAP cov-

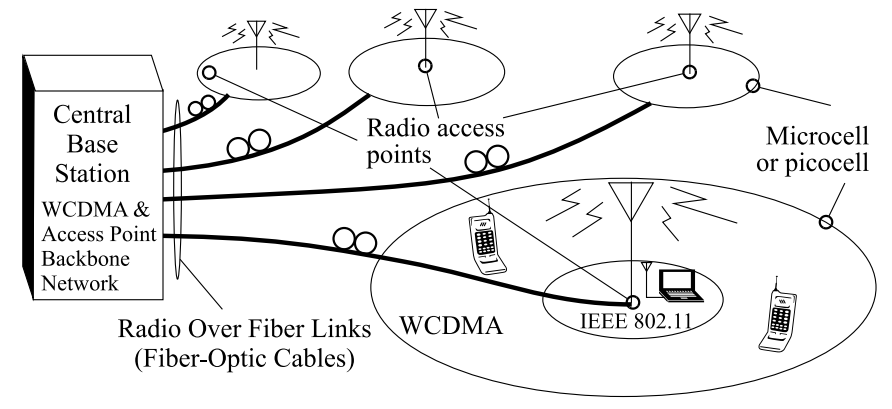

Figure 1: Deployment of microcellular architecture with multiple base stations employing radio over fiber links

ers larger area through cellular WCDMA access, and relatively smaller area through WLAN access. In this paper, the signal to distortion and/or interference and noise ratio (SNR, SDNR and SDINR) of the uplink are derived at various points and the performance is analyzed (see Figure 2).

\section{System Analysis}

The SCM architecture supports both WCDMA and WLAN systems. In the uplink, wideband spread spectrum signals are transmitted from multiple MSs and then they are received at the RAP. The RAP simply converts electrical signals to optical signals and vice versa. Let a subscript $i$ be the $i$ th MS signal of the WCDMA system for $i=1,2, \cdots, n$ where, $n$ is the total number of MS that is active in the system. Let a subscript $\beta$ be an indication for the MS signal that is belonged to the WLAN system. We consider direct sequence spread spectrum (DSSS). Using $P_{s, i}$ as the symbol power of the $i$ th WCDMA signal, the WCDMA signal per symbol that is received at the RAP is given as:

$$
s_{\text {wcdma }}(t)=\sum_{k=-\infty}^{\infty} \sum_{i=1}^{n} \sqrt{2 P_{s, i}} d_{i, k}(t) c_{i}(t) \cos \left(\omega_{\alpha} t\right)
$$

where, $A_{i}$ is the Rayleigh distributed signal amplitude and $A_{i}=\sqrt{2 P_{s, i}}, d_{i, k}(t)$ is the random data sequence, $c_{i}(t)$ is the coded waveform and $\omega_{\alpha}$ is the carrier frequency. Similarly, 


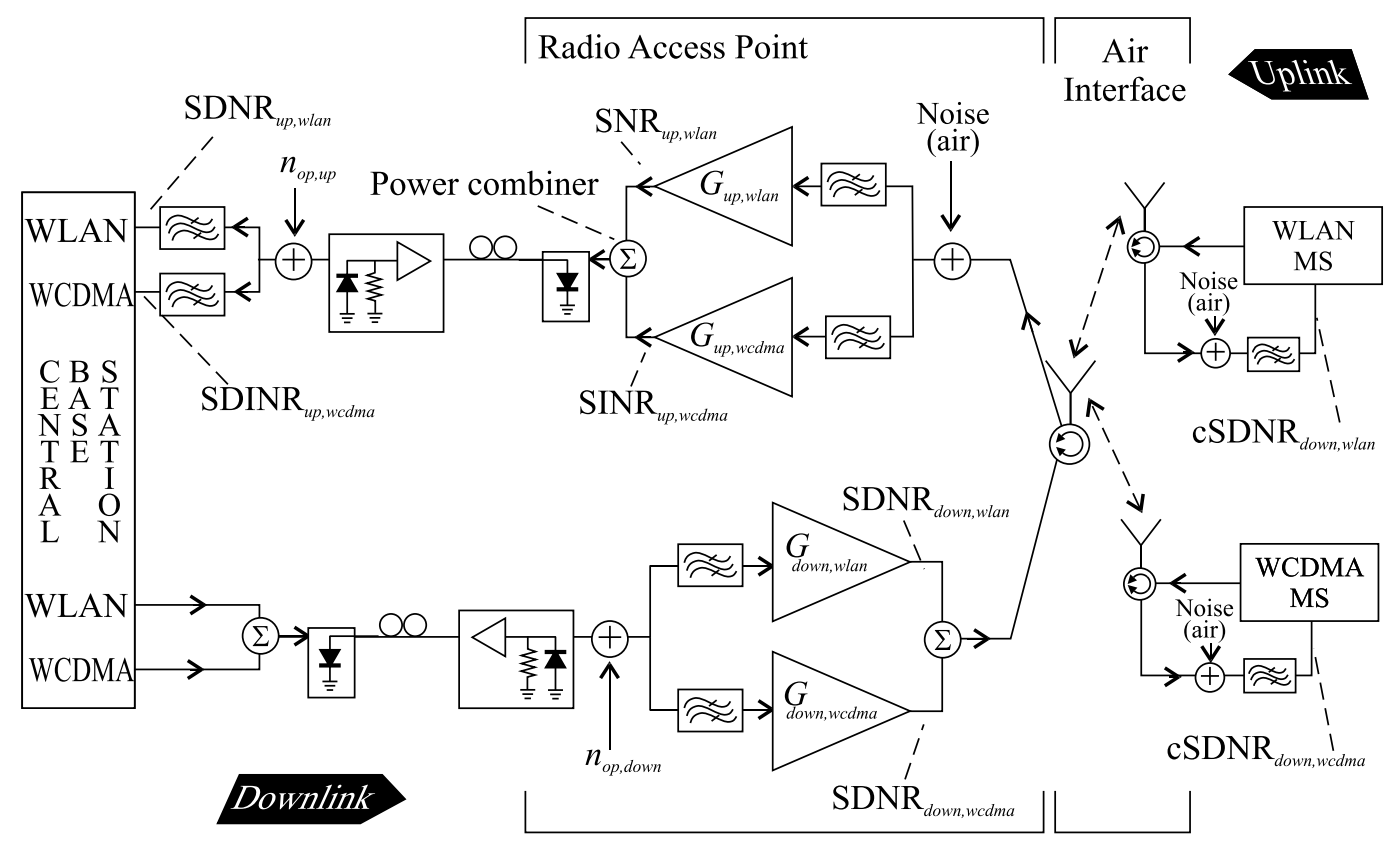

Figure 2: SCM architecture

IEEE 802.11 signal that also employs DSSS has the same form as the WCDMA signal (1).

$$
s_{\text {wlan }}(t)=\sum_{k=-\infty}^{\infty} \sqrt{2 P_{s, \text { wlan }}} d_{\beta, k}(t) c_{\beta}(t) \cos \left(\omega_{\beta} t\right)
$$

where, $P_{s \text {,wlan }}$ is the received symbol power at the RAP, and $B_{\beta}$ is the Rayleigh distributed signal amplitude which $B_{\beta}=$ $\sqrt{2 P_{s, \text { wlan }}}, d_{\beta, k}(t)$ is the data sequence, $c_{\beta}(t)$ is the coded waveform and $\omega_{\beta}$ is the carrier frequency. Expression (2) reflects the fact that there is only one $M S$ can access the radio medium at a time in a WLAN system according to the Carrier Sense Collision Avoidance (CSCA) protocol. At the RAP, the received signal that consists of both WCDMA and WLAN signals is given as:

$$
s_{\mathrm{RAP}}(t)=s_{\mathrm{wcdma}}\left(t-\tau_{i}\right)+s_{\mathrm{wlan}}\left(t-\tau_{\beta}\right)+n_{\mathrm{up}, \mathrm{wl}}(t)
$$

where, $\tau_{i}$ and $\tau_{\beta}$ are the time delay, $\phi_{i}=\omega_{\alpha} \tau_{i}$ and $\phi_{\beta}=\omega_{\beta} \tau_{\beta}$ are the phase of signals which are related to the time delay, and $n_{\text {up,wl }}(t)$ is the additive white Gaussian noise in air with a power spectrum density (PSD) $\Phi_{\text {up, wl }}(\omega)=N_{\text {up ,wl }} / 2$. Both systems experience Rayleigh fading. The MSs access to the WCDMA system asynchronously which results in different access time for each MS. The delay $\tau_{i}$ and $\tau_{\beta}$ are the combination of access time delay and a propagation delay. The latter has a shorter duration. The delays are assumed to have an uniform distribution over $[\pi,-\pi]$.

At the RAP, the signal has already experienced fading, path loss and additive noise in the air interface. This signal goes through two bandpass filters and amplifiers centered at 900
$\mathrm{MHz}$ and $2.4 \mathrm{GHz}$ that also suppress out of band noise and boost the signals. An RF power combiner then combines the signals which, then directly modulates the laser.

The signal to noise ratio (SNR) and the signal to (multiple access) interference and noise ratio (SINR) are evaluated before the power combiner as indicated in Figure 2. The following assumption are made: i) the received power of $i$ th WCDMA and WLAN signals are $P_{s, i}$ and $P_{s, \text { wlan }}$ respectively, ii) each amplifier is assumed to have a noise figure of $F$, and iii) the bandpass filters have bandwidths of $B_{\mathrm{wcdma}}$ and $B_{\text {wlan }}$ for WCDMA and WLAN signals respectively. Then the SINR for the desired WCDMA signal where $i=r$ is:

$$
\mathrm{SINR}_{\mathrm{up}, \mathrm{wcdma}}=\frac{P_{s, r}}{N_{\mathrm{up}, \mathrm{wl}} B_{\mathrm{wcdma}} F+\sum_{\substack{i=1 \\ i \neq r}}^{n} P_{s, i}}
$$

Similarly, the SNR for the WLAN signal is:

$$
\mathrm{SNR}_{\mathrm{up}, \text { wlan }}=\frac{P_{s, \text { wlan }}}{N_{\mathrm{up}, \mathrm{wl}} B_{\mathrm{wlan}} F}
$$

The term $\sum_{\substack{i=1 \\ i \neq r}}^{n} P_{s, i}$ in (4) is the power of the interference due to multiple access in the WCDMA system. That power increases with the number of active users in the system. To avoid the near-far effect, we assume that all the power $P_{s, i}$ received at the RAP are equal. On the contrary, the WLAN system does not have near-far effect because only one user is allowed to transmit 
at a time. Both signal and noise are amplified by the power amplifiers with gain of $G_{\text {up,wcdma }}$ and $G_{\text {up,wlan. Therefore, the }}$ actual signal that modulates is,

$$
\begin{aligned}
s_{\text {laser }}(t)= & \sqrt{G_{\text {up }, \text { wcdma }}}\left[s_{\text {wcdma }}\left(t-\tau_{i}\right)+n_{\text {up }, \text { wcdma }}(t)\right] \\
& +\sqrt{G_{\text {up }, \text { wlan }}}\left[s_{\text {wlan }}\left(t-\tau_{\beta}\right)+n_{\text {up }, \text { wlan }}(t)\right]
\end{aligned}
$$

Then, the bandpass filters at the RAP filter out of band noise. The noise power can be expressed as,

$$
\begin{array}{r}
\left\langle n_{\text {up ,wcdma }}^{2}\right\rangle=N_{\text {up }, \text { wl }} F B_{\text {wcdma }} \\
\left\langle n_{\text {up,wlan }}^{2}\right\rangle=N_{\text {up }, \text { wl }} F B_{\text {wlan }}
\end{array}
$$

In the ROF link, the optical modulation index of a WCDMA signal, $m_{\text {wcdma }, i}$, is defined as $A_{i} \sqrt{G_{\text {up,wcdma }}}$. Similarly, the optical modulation index of a WLAN signal, $m_{\text {wlan }}$, is defined as $B_{\beta} \sqrt{G_{\text {up,wlan }}}$. The two optical modulation indices are also Rayleigh distributed because they are proportional to the square root of the individual RF power. Next, we define a cumulative RMS optical modulation index $\mu$, as the square root of the total $\mathrm{RF}$ power that modulates the laser.

$$
\begin{aligned}
\mu & =\sqrt{G_{\text {up }, \text { wcdma }} \sum_{i=1}^{n} \frac{A_{i}^{2}}{2}+G_{\text {up }, \text { wlan }}\left(\frac{B_{\beta}^{2}}{2}\right)} \\
& =\sqrt{\sum_{i=1}^{n} \frac{m_{\mathrm{wcdma}, i}^{2}}{2}+\frac{m_{\mathrm{wlan}}^{2}}{2}}
\end{aligned}
$$

Considering only one symbol, the signal (6) that modulates the laser can be rewritten as:

$$
\begin{aligned}
s_{\text {laser }}(t)= & \sum_{i=1}^{n} m_{\mathrm{wcdma}, i} d_{i}\left(t-\tau_{i}\right) c_{i}\left(t-\tau_{i}\right) \cos \left(\omega_{\alpha} t-\phi_{i}\right) \\
& +m_{\mathrm{wlan}} d_{\beta}\left(t-\tau_{\beta}\right) c_{\beta}\left(t-\tau_{\beta}\right) \cos \left(\omega_{\beta} t-\phi_{\beta}\right) \\
& +n_{\mathrm{up}, \text { air }}(t)
\end{aligned}
$$

and

$$
\begin{aligned}
n_{\mathrm{up}, \text { air }}(t)= & \sqrt{G_{\mathrm{up}, \mathrm{wcdma}}} n_{\mathrm{up}, \mathrm{wcdma}}(t) \\
& +\sqrt{G_{\mathrm{up}, \text { wlan }}} n_{\mathrm{up}, \text { wlan }}(t)
\end{aligned}
$$

The ROF link noise power for the WCDMA system is $\left\langle n_{\text {op,wcdma }}^{2}\right\rangle$. Similarly the ROF link noise power for the WLAN system is $\left\langle n_{\mathrm{op}, \text { wlan }}^{2}\right\rangle$. If the clipping power spectrum is $S(\omega)$, then the clipped power in the WCDMA band is $\left\langle n_{\mathrm{cl}, \mathrm{wcdma}}^{2}\right\rangle=\int_{\omega_{a}}^{\omega_{b}} S(\omega) \approx S\left(\omega_{\alpha}\right) B_{\text {wcdma }}$ where $\omega_{\alpha}$ and $B_{\text {wcdma }}$ are the carrier frequency and bandwidth of the WCDMA signal. Similarly, the clipped power in the WLAN band is, $\left\langle n_{\mathrm{cl}, \mathrm{wlan}}^{2}\right\rangle \approx S\left(\omega_{\beta}\right) B_{\mathrm{wlan}}$

The photocurrent considering static nonlinearity is expressed as:

$$
\begin{aligned}
i_{\mathrm{D}}(t) & =\frac{1}{\sqrt{L_{\mathrm{op}}}}\left[s_{\text {laser }}(t)+a_{3} s_{\text {laser }}^{3}(t)\right]+n_{\mathrm{op}}(t)+n_{\mathrm{cl}}(t) \\
& \approx \frac{s_{\text {laser }}(t)}{\sqrt{L_{\mathrm{op}}}}+\frac{a_{3}}{\sqrt{L_{\mathrm{op}}}}\left[s_{\text {laser }}(t)-n_{\text {up }, \text { air }}(t)\right]^{3} \\
& +n_{\mathrm{op}}(t)+n_{\mathrm{cl}}(t)
\end{aligned}
$$

where, $L_{\mathrm{op}}$ is the total RF power loss in the ROF link, $s_{\text {laser }}$ is the input signal to the laser that is defined in (10), $n_{\mathrm{op}}(t)$ is the noise in the link, and $n_{\mathrm{cl}}(t)$ is the distortion due to clipping effect which $n_{\mathrm{cl}}(t)=n_{\mathrm{cl} \text {,wcdma }}(t)+n_{\mathrm{cl} \text {, wlan }}(t)$. The total RF power loss in the ROF link is dependent on the fiber length. The DC component and the second order terms are omitted because they will be out of band. According to the cube term of the laser model, the noise from the air interface $n_{\mathrm{up} \text {,air }}(t)$ should also be cubed. However, in reality, the noise is quite small. Therefore, its cubic noise term is ignored.

When (12) is expanded, the desired, distorted, interfering, and noise signals can be easily grouped. The WCDMA and WLAN signals after the bandpass filters are given as follows:

$$
\begin{aligned}
i_{\mathrm{D}, \mathrm{wcdma}}(t)= & D_{\mathrm{wcdma}}(t)+\sum_{i=1}^{7} Z_{i}(t) \\
+ & \sqrt{\frac{G_{\mathrm{up}, \mathrm{wcdma}}}{L_{\mathrm{op}}}} n_{\mathrm{up}, \mathrm{wcdma}}(t) \\
& +n_{\mathrm{op}, \mathrm{wcdma}}(t)+n_{\mathrm{cl}, \mathrm{wcdma}}(t)
\end{aligned}
$$

$$
\begin{aligned}
& i_{\mathrm{D}, \text { wlan }}(t)=D_{\mathrm{wlan}}(t)+\sum_{i=1}^{3} M_{i}(t)+\sqrt{\frac{G_{\mathrm{up}, \mathrm{wlan}}}{L_{\mathrm{op}}}} n_{\mathrm{up}, \text { wlan }}(t) \\
& +n_{\mathrm{op}, \text { wlan }}(t)+n_{\mathrm{cl}, \mathrm{wlan}}(t)
\end{aligned}
$$

where, $i_{\mathrm{D} \text {, wcdma }}(t)$ is the WCDMA signal and $i_{\mathrm{D} \text {, wlan }}(t)$ is the WLAN signal. Finally, the cumulative SDINR of a WCDMA signal and the cumulative SDNR of a WLAN signal are given as:

$$
\begin{aligned}
& \operatorname{SDINR}_{u p, w c d m a}= \\
& \frac{\left\langle D_{\mathrm{wcdma}}^{2}\right\rangle}{\frac{G_{\mathrm{up}, \mathrm{wcdma}}}{L_{\mathrm{op}}}\left\langle n_{\mathrm{up}, \mathrm{wcdma}}^{2}\right\rangle+\left\langle n_{\mathrm{op}, \mathrm{wcdma}}^{2}\right\rangle+\left\langle n_{\mathrm{cl}, \mathrm{wcdma}}^{2}\right\rangle+\sum_{i=1}^{7}\left\langle Z_{i}^{2}\right\rangle}
\end{aligned}
$$




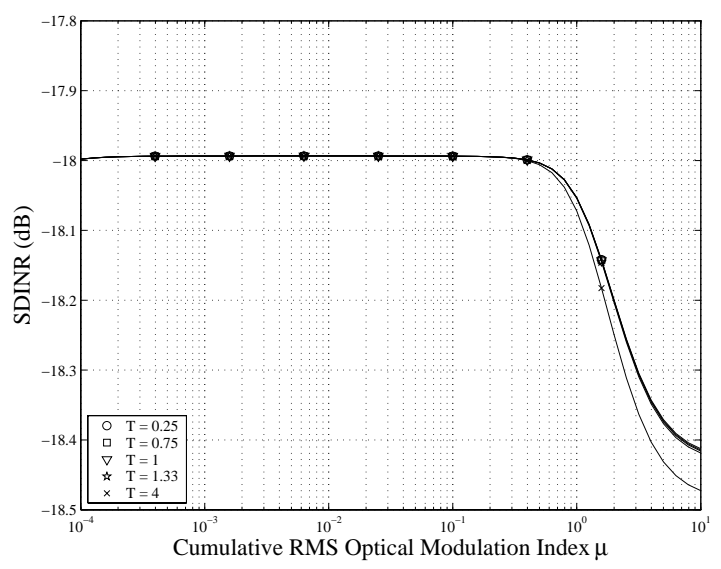

Figure 3: The cumulative SDINR of a WCDMA signal versus the cumulative RMS optical modulation index $\mu$.

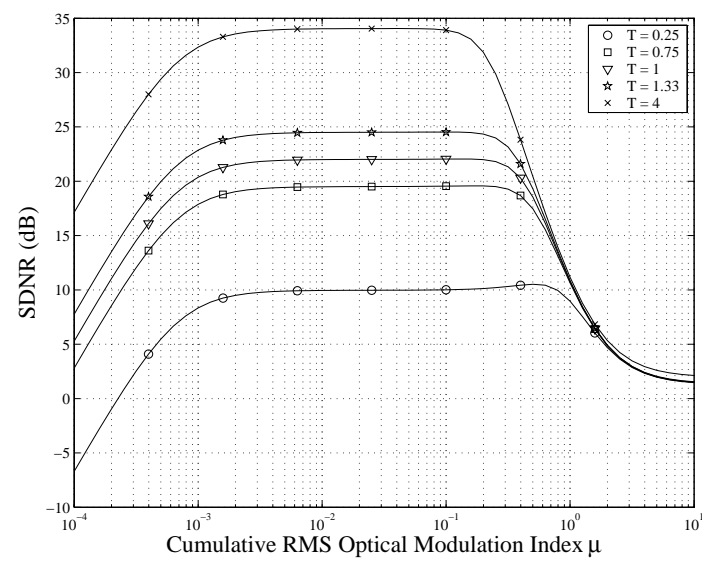

Figure 4: The cumulative SDNR of a WLAN signal versus the cumulative RMS optical modulation index $\mu$.

$$
\begin{aligned}
& \mathrm{SDNR}_{\text {up }, \text { wlan }}= \\
& \frac{\left\langle D_{\mathrm{wlan}}^{2}\right\rangle}{\frac{G_{\mathrm{up}, \mathrm{wlan}}}{L_{\mathrm{op}}}\left\langle n_{\mathrm{up}, \mathrm{wlan}}^{2}\right\rangle+\left\langle n_{\mathrm{op}, \mathrm{wlan}}^{2}\right\rangle+\left\langle n_{\mathrm{cl}, \mathrm{wlan}}^{2}\right\rangle+\sum_{i=1}^{3}\left\langle M_{i}^{2}\right\rangle}
\end{aligned}
$$

where, $\sum_{i=1}^{7}\left\langle Z_{i}^{2}\right\rangle$ and $\sum_{i=1}^{3}\left\langle M_{i}^{2}\right\rangle$ that consists of nonlinear distortion terms that are defined in [5].

\section{Results ANd Discussion}

In the uplink, the signal first goes through the air interface then the ROF link. The cumulative SDINR and SDNR are evaluated at the end of the ROF link. The following assumptions are taken: i) all WCDMA signals at the RAP have equal power; ii) all signals experience the same noise spectrum in the air interface; and iii) an optical modulation index ratio $T$ indicates the power ratio among signals $T=m_{\text {wlan }} / m_{\text {wcdma }, i}$.

In Figure 3, the SDINR is negative because it is evaluate before de-spreading the WCDMA signal. Hence there is no WCDMA processing gain. It is surprising to note that the SDINR of the WCDMA system is not actually affected by the power ratio $T$. This is because of the large spreading gain of the WCDMA system that enable low power operation. When the $\mu>0.6$, nonlinear distortion becomes significant. Similar characteristics can be observed from Figure 4. However, the quality of the WLAN signal changes with $T$. When $T$ is large, a better SDNR is resulted for WLAN and vice versa. The results imply that allocating relatively more power to the WLAN system will improve its performance, provided it meets the ISM band power regulation by FCC. The other fixed parameters in these two figures are: $5 \mathrm{~km}$ ROF link, and 40dB for the $\mathrm{SNR}_{\text {up,wlan }}$.

There is a special reason to select $\mathrm{SNR}_{\text {up,wlan }}$ in Figure 5. The $\mathrm{SNR}_{\text {up,wlan }}$ is the SNR for a WLAN signal at the RAP that really reflects the amount of noise at the air interface (SINR $_{\text {up,wcdma }}$ also includes multi access interference). From the figure, the SDINR does not improve after $20 \mathrm{~dB} \mathrm{SNR}_{\text {up, wlan }}$ at the RAP for WCDMA system. This shows that after this level, the noise in the air interface becomes immaterial and the system is limited by other factors (optical link noise and nonlinear distortion for example). This is different from the WLAN system where, for small $\mu$, the SDNR linearly increases with

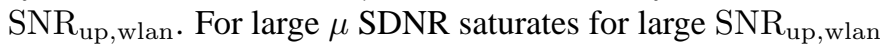
due to nonlinear distortion. In these two figures $T=1$ which means equal power distribution between the WCDMA and WLAN systems, and the length of the ROF link is $10 \mathrm{~km}$.

In Figure 7 and 8, the ratio $T$ indicates the power distribution between the WCDMA and WLAN systems. Large $T$ means more power is allocated to the WLAN system and vice versa. It can be seen that SDINR of WCDMA system is bad irrespective of $T$ when $\mu$ is large (0.8). This is due to excessive nonlinear distortion [6]. For small $\mu$, the SDINR degrades for $T>8$. On the other hand, the SDNR of the WLAN system increases with $T$, except for when $\mu$ is large $(0.8)$ in which case nonlinearity dominates.

\section{Conclusions}

In this paper we have investigated the performance and limitations of an enhanced fiber fed wireless hot spot supporting both IEEE802.11 WLAN and cellular WCDMA services. We considered subcarrier multiplexing which is the most natural way of supporting these two (frequency separated) systems. Our detailed analysis shows that it is possible to support both systems. However, there should be number considerations before designing such a system with good performance.

1. Relatively more power could be allocated to WLAN system, compared to WCDMA system, without significantly degrading WCDMA system performance. In fact it is better to allocate the maximum power to WLAN allowed by FCC ISM band regulations.

2. The cumulative optical modulation index should be kept 


\begin{tabular}{|c|l|c|}
\hline$n$ & Number of active MSs in WCDMA system & 64 \\
\hline$G_{\text {up,wcdma }}$ & RF power gain for WCDMA signal & $20 \mathrm{~dB}$ \\
\hline$G_{\text {up,wlan }}$ & RF power gain for WLAN signal & $20 \mathrm{~dB}$ \\
\hline$F$ & RAP/Receiver amplifier noise factor & $1 \mathrm{~dB}$ \\
\hline$B_{\text {wcdma }}$ & Bandwidth of WCDMA & $5 \mathrm{MHz}$ \\
\hline$B_{\text {wlan }}$ & Bandwidth of WLAN & $22 \mathrm{MHz}$ \\
\hline$P_{o}$ & Laser mean optical power & $1 \mathrm{~mW}$ \\
\hline$G_{m}$ & Laser modulation gain & $0.12 \mathrm{~A} / \mathrm{W}$ \\
\hline$\Re$ & Photo diode responsivity & $0.75 \mathrm{~W} / \mathrm{A}$ \\
\hline$n_{c}$ & Number of optical connectors & 2 \\
\hline$l_{c}$ & Optical connector loss & $1 \mathrm{~dB}$ \\
\hline$\alpha$ & Fiber attenuation & $0.5 \mathrm{~dB} / \mathrm{km}$ \\
\hline RIN & Relative intensity noise parameter & $-155 \mathrm{~dB} / \mathrm{Hz}$ \\
\hline$T_{o}$ & Optical receiver temperature & $275 \mathrm{~K}$ \\
\hline$R_{L}$ & Receiver load resistance & $50 \Omega$ \\
\hline$a_{3}$ & Third-order nonlinearity parameter & $1 / 3$ \\
\hline
\end{tabular}

Table 1: Parameters used to determine the numerical results; these obtained either from the corresponding standards, manufacturers specification or typical values

below at least 0.6 (may vary depending on particular link parameters), to avoid extensive nonlinear distortion.

3. The overall system performance also depends on fiber length, optical-electrical conversion losses and nonlinear distortion of the laser diode.

These are the major observations, other observations are described in detail under 'Results and Discussion' Section. Note that the numerical values used for simulation are obtained from typical device specifications or from the values we found in our experimental test bed [7] or reported by others; bandwidths depends on bit rates. General trend of the system behavior will not vary much based on these values, however, specific values (such as $\mu<0.6$ will vary.

\section{REFERENCES}

[1] Yang Xiao and J. Rosdahl, "Throughput and delay limits of ieee 802.11," Communications Letters, IEEE, vol. 6, no. 8, pp. $355-357$, Aug. 2002.

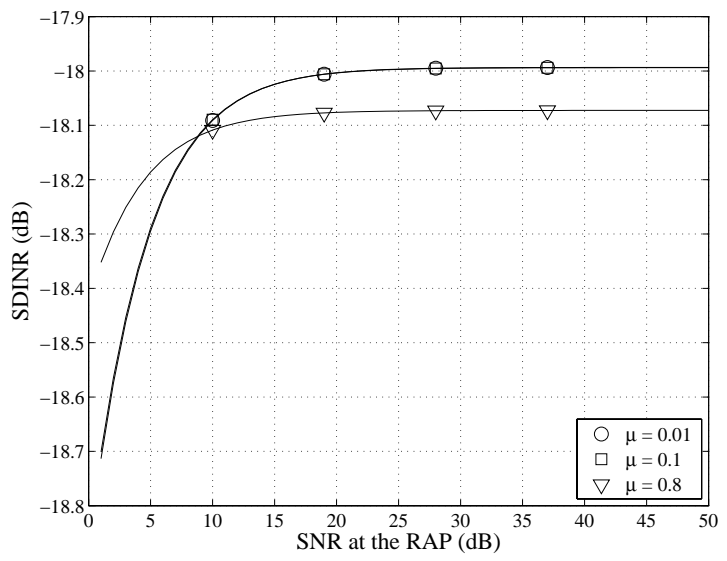

Figure 5: The cumulative SDINR of a WCDMA signal versus the $\mathrm{SNR}_{\text {up, wlan }}$ of WLAN at the RAP.
[2] Roland Yuen and Xavier Fernando, "Analysis of sub-carrier multiplexed radio over fiber link for the simultaneous support of wlan and wcdma systems," Kluwer Wireless Personal Communications Journal, vol. 33, no. 1, pp. 1-20, April 2005.

[3] Xavier Fernando, Hatice Kosek, Yeefeng He, and Xijia Gu, "Optical domain demultiplexing of subcarrier multiplexed cellular and wireless lan radio signals," in Proceedings of the SPIE,. 2005, Photnic North, Microwave Photonic Symposium.

[4] R. Sabella, "Performance analysis of wireless broadband systems employing optical fiber links," Communications, IEEE Transactions on, vol. 47, no. 5, pp. 715 - 721, May 1999.

[5] Roland Yuen, "Investigation of sub carrier multiplexed fiber optic link supporting wlan and wcdma," M.S. thesis, Ryerson University, 2005.

[6] W. I. Way, "Subcarrier multiplexed lightwave system design considerations for subscriber loop applications," Journal of Lightwave Technology, vol. 7, no. 11, pp. 1806-1818, November 1989.

[7] Xavier Fernando and Abu Sesay, "Higher order adaptive filter characterization of microwave fiber optic link nonlinearity," in Proceedings of the SPIE, The International Society of for Optical Engineering, Photonic East, San Jose, January 2000, vol. 3927-06, pp. 39-49.

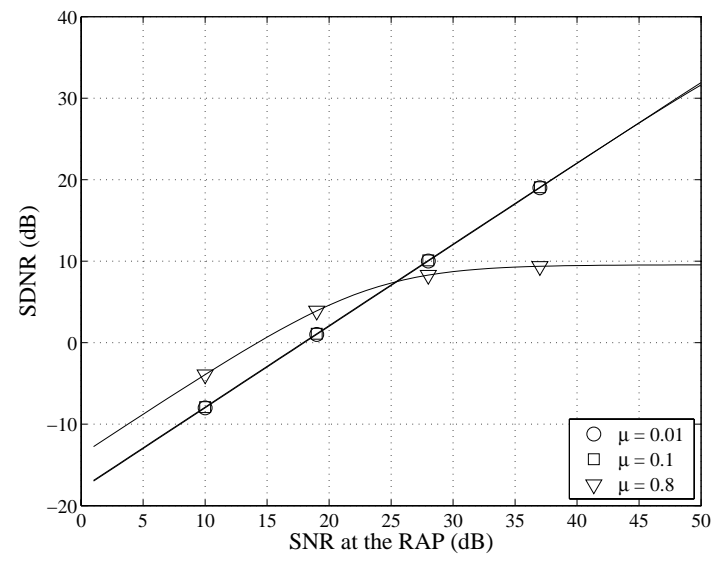

Figure 6: The cumulative SDNR of a WLAN signal versus the $\mathrm{SNR}_{\text {up, wlan }}$ of WLAN at the RAP 


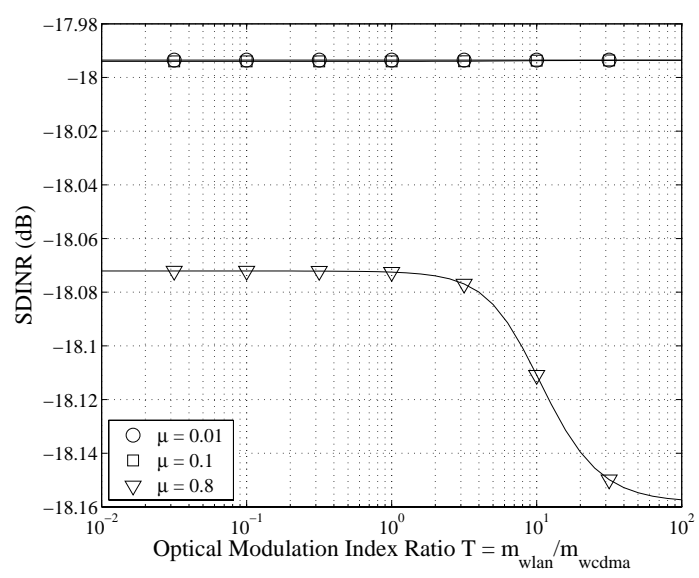

Figure 7: The cumulative SDINR of a WCDMA signal versus the optical modulation index ratios $T$. The $\mathrm{SNR}_{\mathrm{up}, \text { wlan }}$ is $40 \mathrm{~dB}$, and the length of the ROF link is $10 \mathrm{~km}$

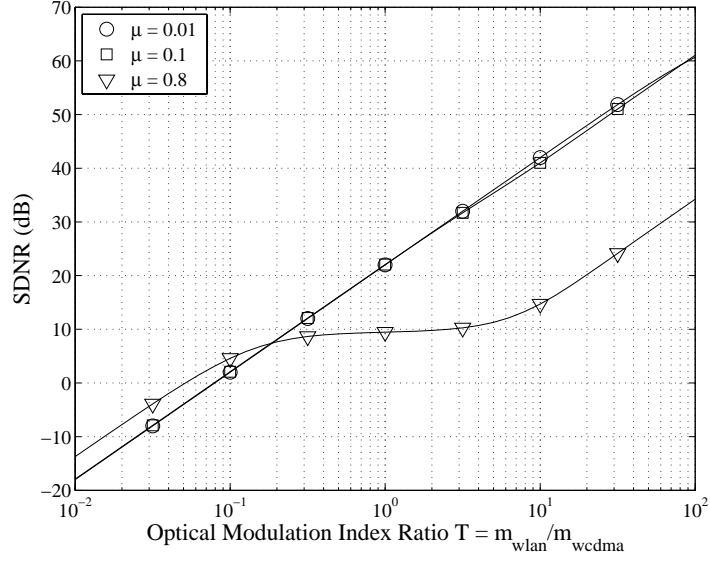

Figure 8: The cumulative SDNR of a WLAN signal versus the optical modulation index ratios $T$. The $\mathrm{SNR}_{\mathrm{up} \text {, wlan }}$ is $40 \mathrm{~dB}$, and the length of the ROF link is $10 \mathrm{~km}$. 